\title{
Squark/gluino searches in hadronic channels with CMS
}

\section{Tai Sakuma for the CMS collaboration}

University of Bristol

E-mail: tai.sakuma@cern.ch

\begin{abstract}
These proceedings summarize the results of four analyses which searched for squarks and gluinos in hadronic final states with missing transverse momentum in $2.3 \mathrm{fb}^{-1}$ of data in proton-proton collisions at $\sqrt{s}=13 \mathrm{TeV}$ collected in the year 2015 with the CMS detector at the CERN LHC. Each analysis is characterized by a different kinematic variable that is sensitive to the presence of invisible particles, e.g., $M_{\mathrm{T} 2}, \alpha_{\mathrm{T}}$, and razor variables. We observed no significant deviation from the standard model prediction and placed limits on the production cross sections and the masses of squarks and gluinos in simplified models of supersymmetric models. The limits are significantly extended from the previous results.
\end{abstract}

Fourth Annual Large Hadron Collider Physics

13-18 June 2016

Lund, Sweden 


\section{Introduction}

The standard model (SM) of particle physics is considered to be incomplete. A main reason is its fine-tuning, by which the very large value of the bare Higgs boson mass must be extremely precisely larger than another very large value of the cutoff of the calculation of higher-order corrections, which, for example, is of the order of the GUT scale $\left(10^{16} \mathrm{GeV}\right)$, by a small value of the physical mass of the Higgs boson $(125 \mathrm{GeV})$. Supersymmetry (SUSY), an extension of the spacetime symmetry which must be broken if it is a symmetry of nature, provides a solution to this problem. The SM can be extended to have a broken SUSY so as to alleviate the dependency of the Higgs boson mass correction on the cutoff from quadratic to logarithmic and reduce the fine-tuning to a level that can be regarded as natural.

Supersymmetric extensions of the SM predict that each particle in the SM has a heavier partner (superpartner) that has not been observed. For the level of the tuning to be natural, some of the superpartners, such as top squarks, bottom squarks, and gluinos, must have masses of the order of $\mathrm{TeV}$ and can be produced in proton-proton (pp) collisions at the CERN LHC. Squark pairs and gluino pairs have larger production cross sections than other superpartners and have jets and a large missing transverse momentum in the final states of their decay products. Therefore, squark and gluino searches in hadronic channels are optimum for early searches in LHC Run 2.

These proceedings summarize the results of four analyses [1-4] that searched for squarks and gluinos in hadronic final states with a large missing transverse momentum in $2.3 \mathrm{fb}^{-1}$ of data in pp collisions at $\sqrt{s}=13 \mathrm{TeV}$ collected with the CMS detector in 2015, the first year of LHC Run 2. Each analysis is characterized by a different kinematic variable used in the analysis, e.g., $M_{\mathrm{T} 2}[5,6]$, $\alpha_{\mathrm{T}}$ [7], and razor variables [8]. We found no evidence of SUSY and placed limits on the production cross sections and the masses of squarks and gluinos in simplified models [9-14] of SUSY models.

\section{Overall search procedure}

After introducing common kinematic variables used in the searches, this section reviews an overall search procedure of SUSY searches in hadronic final states.

The scalar sum of the transverse momenta $\left(p_{\mathrm{T}}\right)$ of the jets, denoted by $H_{\mathrm{T}}$, is a measure of the energy scale of the event. The missing transverse momentum, which is sensitive to the presence of invisible particles and a measure of their total transverse momentum, is the negative of the vector sum of the $\vec{p}_{\mathrm{T}}$ of all reconstructed particles, $\vec{E}_{\mathrm{T}}^{\text {miss }}$, or its magnitude, $E_{\mathrm{T}}^{\text {miss }}$. Alternatively, $\vec{H}_{\mathrm{T}}^{\text {miss }}$ and $H_{\mathrm{T}}^{\text {miss }}$, which are, respectively, the negative of the vector sum of the $\vec{p}_{\mathrm{T}}$ of the jets and its magnitude, are also used. The azimuthal angle between a jet $\vec{p}_{\mathrm{T}}$ and $\vec{H}_{\mathrm{T}}^{\text {miss }}$ (or $\vec{E}_{\mathrm{T}}^{\text {miss }}$ ), written as $\Delta \phi$, is used to suppress QCD multijet events. The number of the jets in the event is denoted by $n_{\text {jet }}$. The number of the jets which are tagged as originating from the bottom quark is denoted by $n_{\mathrm{b}}$.

We start a search by defining a search (signal) region, a region of the phase space in which we search for a signal as an excess of the number of the events from the SM prediction. Search regions are specified in terms of common variables described in the previous paragraph, i.e., $H_{\mathrm{T}}, n_{\mathrm{jet}}, H_{\mathrm{T}}^{\text {miss }}$, $E_{\mathrm{T}}^{\mathrm{miss}}$, and $\Delta \phi$, or special variables such as $M_{\mathrm{T} 2}, \alpha_{\mathrm{T}}, \Delta \phi_{\min }^{*}$ and razor variables, introduced in later sections. Events in the search regions are required not to have a isolated charged lepton or photon (vetos). Dominant SM background processes in search regions are $\mathrm{Z}(\rightarrow v \bar{v})+\mathrm{jets}, \mathrm{W}+\mathrm{jets}, \mathrm{t} \overline{\mathrm{t}}$, and 
QCD multijets events. We perform a blind analysis, by which we do not analyze the data in the search region until later in the procedure.

We, then, define control regions (CRs), for example, by inverting vetos or as sidebands in variables used to define the search region. CRs are used to predict the background in the search region from the same or similar SM processes that dominate the CRs. For example, double-lepton $\mathrm{CRs}$, in which an event is required to have jets and a pair of electrons or muons, are dominated by the $\mathrm{Z}\left(\rightarrow \ell^{+} \ell^{-}\right)+$jets events, where $\ell=\mathrm{e}, \mu$. A single-photon $\mathrm{CR}$, in which an event has jets and a single isolated photon, is dominated by the $\gamma+$ jets events. These CRs are used to predict the background from the $\mathrm{Z}(\rightarrow v \bar{v})+$ jets events. Single-lepton CRs, which have jets and single isolated electrons or muons, are dominated by the $\mathrm{W}+\mathrm{jets}$ and $\mathrm{t} \overline{\mathrm{t}}$ events and are used to predict the background from these processes. A sideband of a search region in $\Delta \phi$, which is dominated by QCD multijets events, can be used to predict the QCD multijets background.

We define categories, i.e., events in the signal region are categorized, for example, in bins of $H_{\mathrm{T}}, n_{\mathrm{jet}}$, and $n_{\mathrm{b}}$. By using the data in CRs and simulated events in various methods, we predict the background in each category in the search region and validate the prediction.

After the background predictions are validated, we, finally, analyze the data in the search region (unblinding) and compare, in each category, with the predictions. Unless we observe a significant discrepancy in the comparison, we interpret the results in simplified models and place upper limits on the production cross sections of superpartners in the models with the CLs method $[15,16]$ with the asymptotic formula [17] and exclusion limits on the masses of superpartners in the models by comparing with the theoretical cross sections.

\section{Search with $H_{\mathrm{T}}^{\text {miss }}$}

This section summarizes the analysis published in Ref. [1], the first publication of the CMS SUSY searches with the Run 2 data. The analysis searches high- $H_{\mathrm{T}}$ events with four or more jets with no isolated electron, muon, or track. It uses a combination of two search strategies used in Run $1[18,19]$.

The search region is specified as $n_{\mathrm{jet}} \geq 4, H_{\mathrm{T}}>500 \mathrm{GeV}, H_{\mathrm{T}}^{\text {miss }}>200 \mathrm{GeV}$, and $\Delta \phi>0.5(>0.3)$ for the two largest $p_{\mathrm{T}}$ jets (the $3^{\text {rd }}$ and $4^{\text {th }}$ largest $p_{\mathrm{T}}$ jets). The events in the search region are categorized in the bins for $H_{\mathrm{T}}, H_{\mathrm{T}}^{\text {miss }}, n_{\mathrm{jet}}$, and $n_{\mathrm{b}}$. The bin boundaries in $H_{\mathrm{T}}$ and $H_{\mathrm{T}}^{\text {miss }}$ are illustrated in Fig. 1 . The intervals of the $n_{\text {jet }}$ bins are 4-6, 7-8, $\geq 9$, and those for the $n_{\mathrm{b}}$ bins are $0,1,2, \geq 3$. The analysis total has 72 bins.

The pie charts in Fig. 2 show the background composition of the SM processes in the bins of $n_{\text {jet }}$ and $n_{\mathrm{b}}$ evaluated in simulation. The composition noticeably changes with $n_{\text {jet }}$ and $n_{\mathrm{b}}$. For example, the lowest $n_{\text {jet }}-n_{\mathrm{b}}$ bin contains an approximately even mixture of the $\mathrm{Z}(\rightarrow v \bar{v})+\mathrm{jets}, \mathrm{W}+\mathrm{jets}$, and QCD multijets events and a small fraction of the $\bar{t} \bar{t}$ events while the highest $n_{\text {jet }}-n_{\mathrm{b}}$ bin is dominated by the t⿱亠t events.

The background from the $\mathrm{W}+\mathrm{jets}$ and $\mathrm{t} \overline{\mathrm{t}}$ events are divided into two groups: the lost-lepton background, in which the electron or muon from the $\mathrm{W}$ boson decay does not cause a veto, and the hadronically decaying $\tau$ background, in which the $\tau$ lepton from the $\mathrm{W}$ boson decay hadronically decays. The background predictions are separately made for these two groups as well as for the 


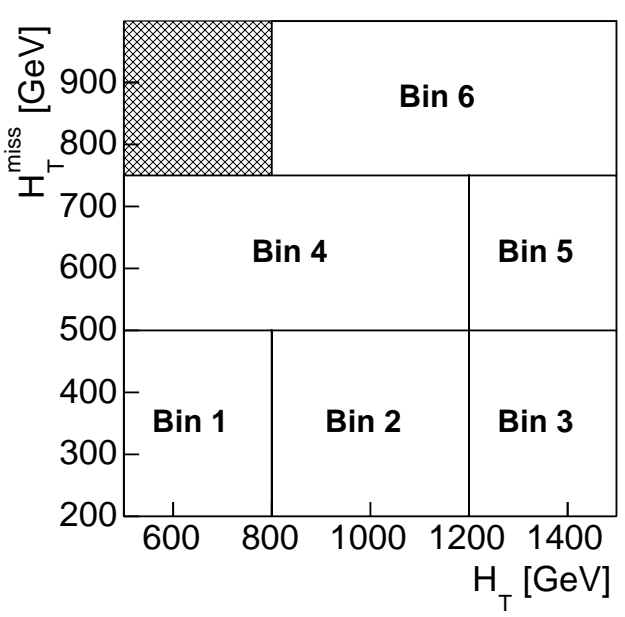

Figure 1: A schematic diagram of the categories used in Ref. [1], i.e., bins in $H_{\mathrm{T}}$ and $H_{\mathrm{T}}^{\text {miss }}$. Events in each bin in the diagram are further binned in $n_{\text {jet }}$ and $n_{\mathrm{b}}$.

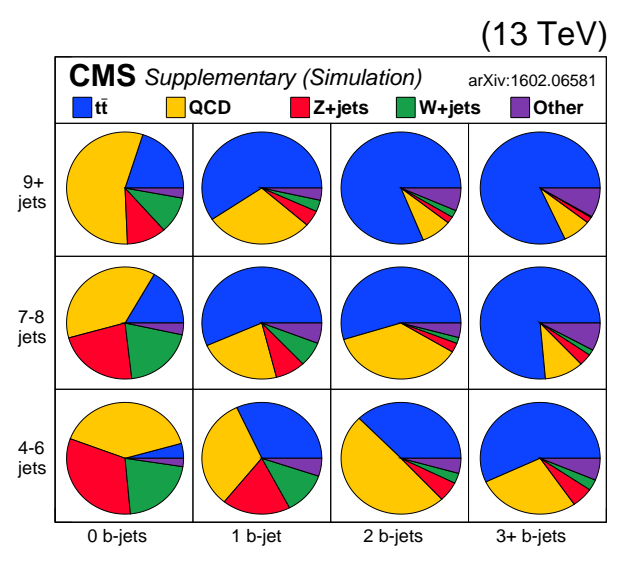

Figure 2: Background composition of the SM processes in the bins of $n_{\text {jet }}$ and $n_{\mathrm{b}}$ evaluated in simulation in the analysis in Ref. [1].

$\mathrm{Z}(\rightarrow v \bar{v})+$ jets events and the QCD multijet events. In addition to the details of the background predictions, the evaluation of the impact of signal contamination in CRs is described in Ref. [1].

The observed number of the events and the SM background predictions in each of the 72 bins in the search region is shown in Fig. 3. The numerical values are given in Ref. [1]. The observed numbers are consistent with the predictions. The interpretations of the results are summarized in Section 7.

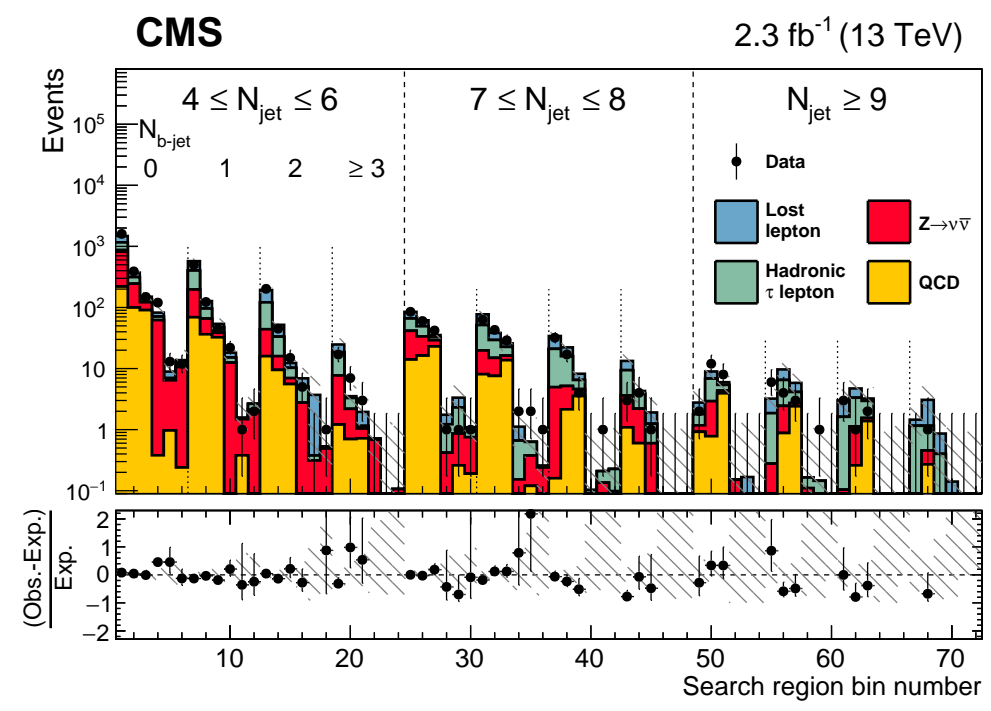

Figure 3: The observed number of the events and the SM background predictions in each of the 72 bins in the search region of the analysis in Ref. [1]. 


\section{Search with $M_{\mathrm{T} 2}$}

In the analysis described in Ref. [2], the $M_{\mathrm{T} 2}$ variable is used to define the search region and categories. This variable was also used in searches in hadronic final states in Run 1 [20, 21].

The $M_{\mathrm{T} 2}$ variable is an extension of the transverse mass to the case in which the event has two invisible particles in the final state $[5,6]$. It is defined as

$$
M_{\mathrm{T} 2}=\min _{\vec{q}_{\mathrm{T}}+\vec{r}_{\mathrm{T}}=\vec{E}_{\mathrm{T}}^{\text {miss }}}\left[\max \left(M_{\mathrm{T}}\left(\vec{p}_{\mathrm{T}}^{j_{1}}, \vec{q}_{\mathrm{T}}\right), M_{\mathrm{T}}\left(\vec{p}_{\mathrm{T}}^{j_{2}}, \vec{r}_{\mathrm{T}}\right)\right)\right]
$$

where $\vec{p}_{\mathrm{T}}^{j_{1}}$ and $\vec{p}_{\mathrm{T}}^{j_{2}}$ are $p_{\mathrm{T}}$ of two pseudo-jets, sets of jets formed to maximize their invariant mass, and $M_{\mathrm{T}}$ is the transverse mass, i.e., $M_{\mathrm{T}}\left(\vec{p}_{\mathrm{T}}, \vec{q}_{\mathrm{T}}\right)=\sqrt{2\left(\left|\vec{p}_{\mathrm{T}}\right|\left|\vec{q}_{\mathrm{T}}\right|-\vec{p}_{\mathrm{T}} \cdot \vec{q}_{\mathrm{T}}\right)}$. In words, among the variations of two components into which $\vec{E}_{\mathrm{T}}^{\text {miss }}$ can be split, $M_{\mathrm{T} 2}$ is the minimum value of the larger value of the two transverse masses which are formed from two pairs of a pseudo-jet and one of the two $\vec{E}_{\mathrm{T}}^{\text {miss }}$ components. As can be seen in Fig. 4, while $M_{\mathrm{T} 2}$ is small for QCD multijet events, it can be large for signal events.

In contrast to the Run 1 analyses [20, 21], the search region in this analysis is extended to include events with one jet (monojet). Therefore, the events in the search region have one or more jets and no isolated electron, muon, or track. Because of the conditions of the online triggers used in the analysis, the events in the search region satisfies either $H_{\mathrm{T}}>200 \mathrm{GeV}$ and $E_{\mathrm{T}}^{\text {miss }}>200 \mathrm{GeV}$ or $H_{\mathrm{T}}>1000 \mathrm{GeV}$ and $E_{\mathrm{T}}^{\text {miss }}>30 \mathrm{GeV}$. Further, the events with two or more jets are required to have $M_{\mathrm{T} 2}>200 \mathrm{GeV}, \Delta \phi>0.3$ with $E_{\mathrm{T}}^{\text {miss }}$ for the four largest $p_{\mathrm{T}}$ jets, and $\left|E_{\mathrm{T}}^{\text {miss }}-H_{\mathrm{T}}^{\text {miss }}\right| / E_{\mathrm{T}}^{\text {miss }}<0.5$.

The events in the search region are categorized in the five $H_{\mathrm{T}}$ bins and eleven $n_{\text {jet }}$ and $n_{\mathrm{b}}$ bins. The events with two ore more jets are further categorized in the bins of $M_{\mathrm{T} 2}$. The boundaries of the $M_{\mathrm{T} 2}$ bins depend on the $H_{\mathrm{T}}$ bin and are illustrated on the top in Fig. 5. The monojet events are

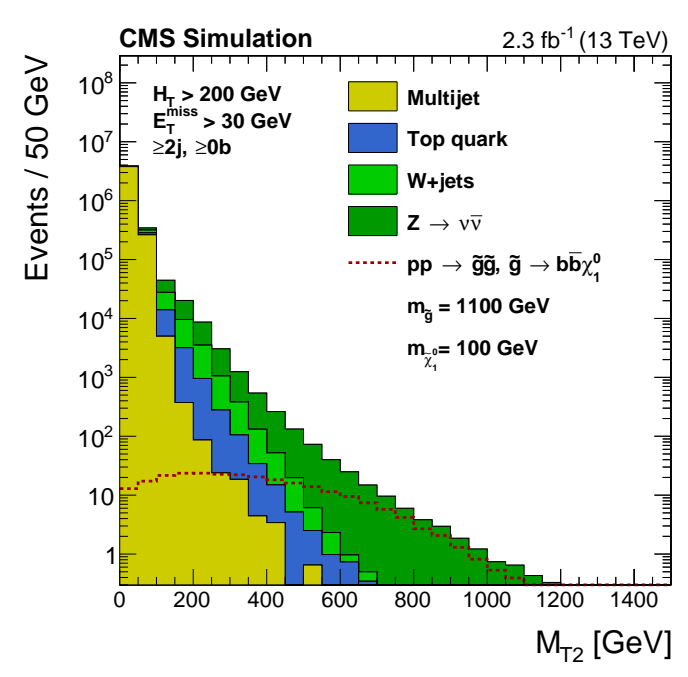

Figure 4: Distributions of $M_{\mathrm{T} 2}$ in simulated events for the SM background processes and a signal process [2].

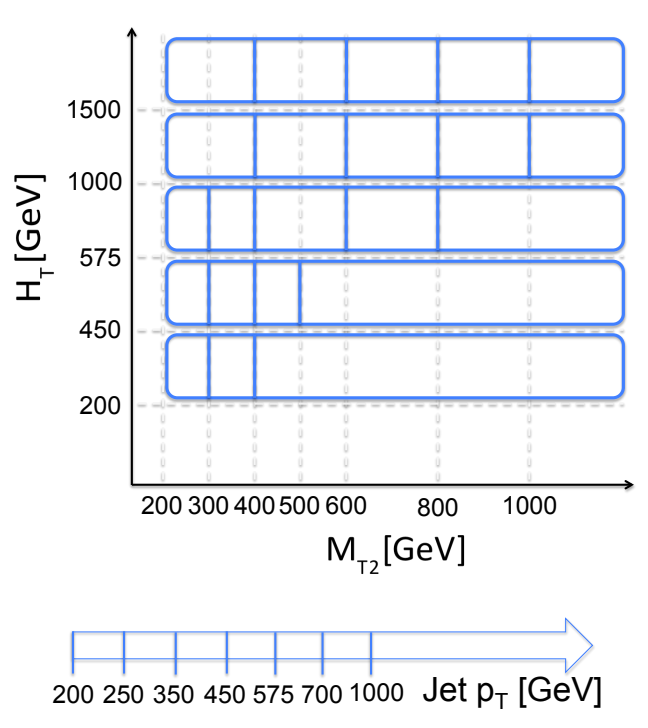

Figure 5: (top) The boundaries of the $H_{\mathrm{T}}$ and $M_{\mathrm{T} 2}$ bins for $n_{\text {jet }} \geq 2$ and (bottom) the boundaries of the jet $p_{\mathrm{T}}$ bins for $n_{\text {jet }}=1$ in Ref. [2]. 


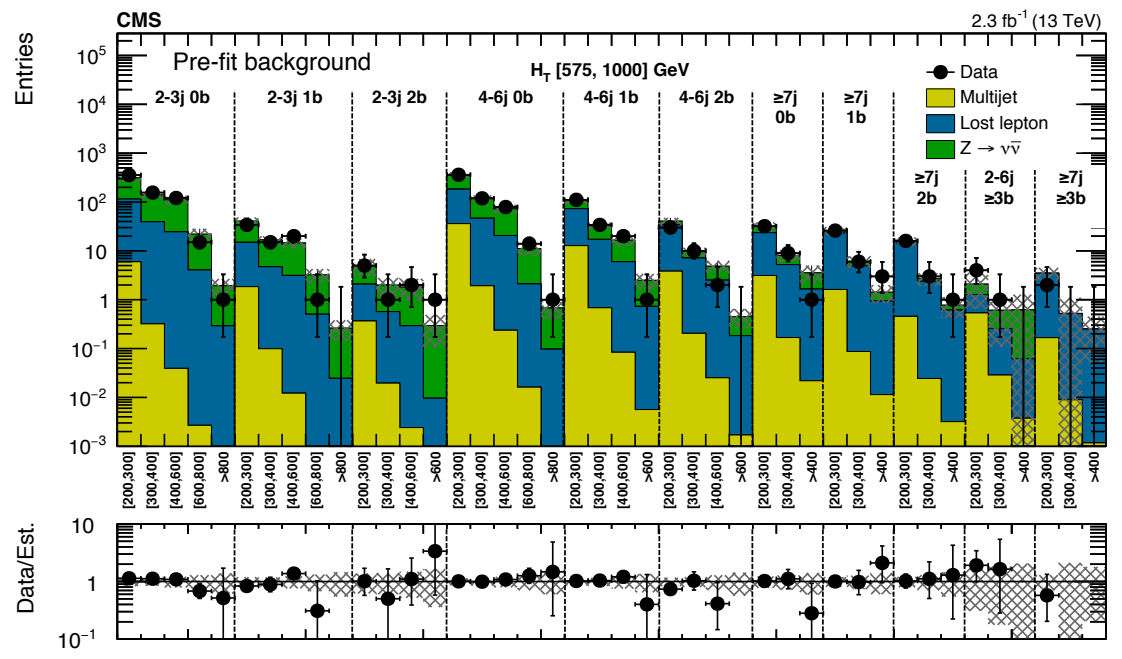

Figure 6: The observed number of the events and the SM background predictions in the $n_{\text {jet }}, n_{\mathrm{b}}$, and $M_{\mathrm{T} 2}$ bins in one of the $H_{\mathrm{T}}$ bins in the search region of the analysis in Ref. [2].

categorized in the bins of the jet $p_{\mathrm{T}}$, as shown on the bottom in Fig. 5. The analysis total has 172 categories.

The SM background is predicted for the QCD multijet, lost-lepton, and $\mathrm{Z}(\rightarrow v \bar{v})+$ jets events. The data are consistent with the predictions. Figure 6 shows the comparison in the $n_{\mathrm{jet}}, n_{\mathrm{b}}$, and $M_{\mathrm{T} 2}$ bins in one of the $H_{\mathrm{T}}$ bins. Similar figures for the other bins are shown in Ref. [2]. In addition, Ref. [2] provides the numerical values of the the predictions and observations in 14 aggravated bins for simpler reinterpretations. Section 7 includes a summary of the interpretations of the results.

\section{Search with razor variables}

The analysis reported in Ref. [3] uses the razor variables in the definition of the search region and the background prediction. The razor variables were used in SUSY searches in Run 1 [22-27]. The analysis searches events with zero or one lepton, and four or more jets.

In defining the razor variables, longitudinal momenta are used to find an approximate center-of-mass frame of the patron-level process [8]. For events in SUSY models, the distribution of the razor variable $M_{\mathrm{T}}^{\mathrm{R}}$, with the dimension of mass, has a broad peak, and the distribution of the dimensionless razor variable $R^{2}$ has a long

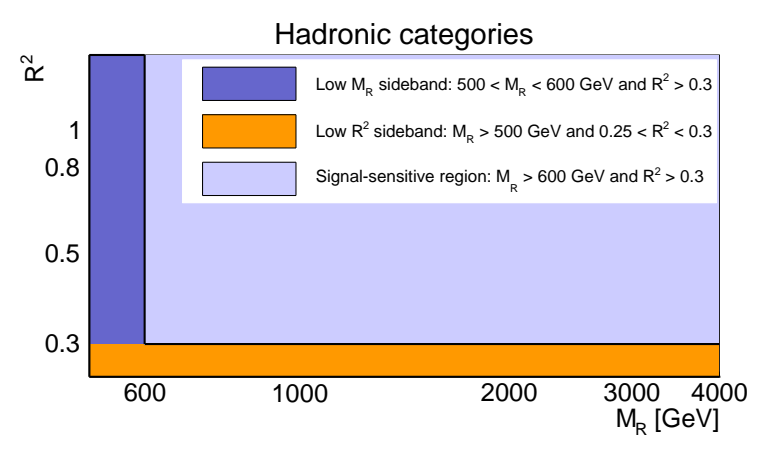

Figure 7: The sidebands and the signal region on the $M_{\mathrm{T}}^{\mathrm{R}}-R^{2}$ plane in the multijet category in Ref. [3] tail. In contrast, for the events in the SM processes, the distributions of both variables exponentially decrease, which is used to model the background distribution on the $M_{\mathrm{T}}^{\mathrm{R}}-R^{2}$ plane. 
The analysis defines three event categories in the search region based on the presence of an electron or muon. The events in the muon multijet (electron multijet) category are required to have one muon with $p_{\mathrm{T}}>20 \mathrm{GeV}$ (one electron with $p_{\mathrm{T}}>25 \mathrm{GeV}$ ), $M_{\mathrm{T}}>120 \mathrm{GeV}, 4$ jets with $p_{\mathrm{T}}>$ $40 \mathrm{GeV}, M_{\mathrm{T}}^{\mathrm{R}}>400 \mathrm{GeV}$, and $R^{2}>0.15$. The events in the multijet category has 4 jets with $p_{\mathrm{T}}>$ $40 \mathrm{GeV}$ or 2 jets with $p_{\mathrm{T}}>80 \mathrm{GeV}, M_{\mathrm{T}}^{\mathrm{R}}>500 \mathrm{GeV}$, and $R^{2}>0.25$. The events in the muon multijet and electron multijet categories (multijet category) are further categorized in 56 (35) bins of $M_{\mathrm{T}}^{\mathrm{R}}$ and $R^{2}$ and $4 n_{\mathrm{b}}$ bins.

A function of $M_{\mathrm{T}}^{\mathrm{R}}$ and $R^{2}$ with two additional parameters is used to model the dependency of the distributions of the SM background events on $M_{\mathrm{T}}^{\mathrm{R}}$ and $R^{2}$. This function, which is fit in each $n_{\mathrm{b}}$ bin to the data in sidebands of the signal region in $M_{\mathrm{T}}^{\mathrm{R}}$ and $R^{2}$, models the distributions of the background events in the bins of $M_{\mathrm{T}}^{\mathrm{R}}$ and $R^{2}$. The sidebands on the $M_{\mathrm{T}}^{\mathrm{R}}-R^{2}$ plane are illustrated in Fig. 7. No significant deviations in the data from the prediction is observed. Figures showing the comparisons of the data and the predictions for each bin are provided in Ref. [3]. A summary of the interpretations is given in Section 7.

\section{Search with $\alpha_{\mathrm{T}}$}

The analysis reported in Ref. [4] uses the variables $\alpha_{\mathrm{T}}$ and $\Delta \phi_{\min }^{*}$ to suppress the QCD multijet background to a negligible level. These variables were used in SUSY searches in Run 1 [7, 28-31].

The $\alpha_{\mathrm{T}}$ variable [7] is defined as

$$
\alpha_{\mathrm{T}}=\frac{\sum_{i \in \text { jets }} E_{\mathrm{T}}^{i}-\Delta E_{\mathrm{T}}}{2 \sqrt{\left(\sum_{i \in \text { jets }} E_{\mathrm{T}}^{i}\right)^{2}-H_{\mathrm{T}}^{\text {miss }}}},
$$

where $E_{\mathrm{T}}^{i}\left(\equiv E^{i} \sin \theta^{i}\right)$ is the jet transverse energy, and $\Delta E_{\mathrm{T}}$ is the difference in $E_{\mathrm{T}}$ of the two pseudo-jets, two sets of jets into which the jets in the event are combined so as to minimize $\Delta E_{\mathrm{T}}$. As can be seen in Fig. 8, while the value of $\alpha_{\mathrm{T}}$ for QCD multijet events do not become much larger than 0.5 , it can be for events from the other processes. The $\Delta \phi_{\min }^{*}$ is the minimum of the azimuthal angle between a jet and $H_{\mathrm{T}}^{\text {miss }}$ recomputed without the jet, i.e.,

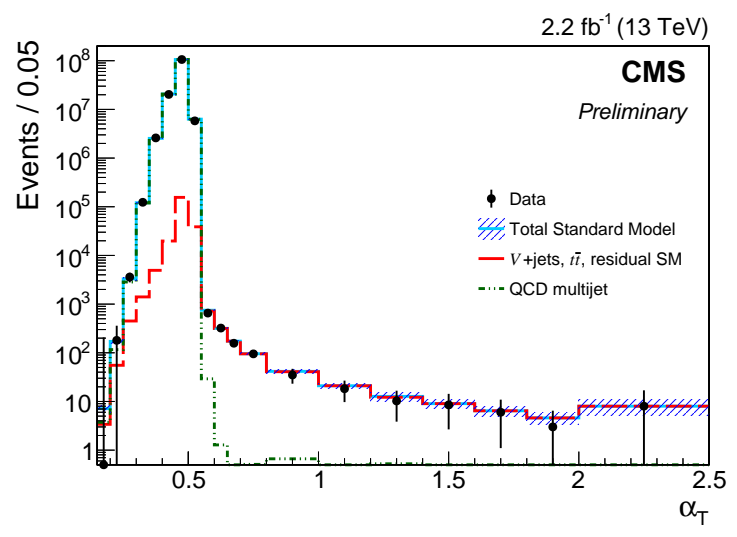

Figure 8: The distribution of $\alpha_{\mathrm{T}}$ in the data and estimates of the contributions from the QCD multijet events and the other SM events [4].

$$
\Delta \phi_{\min }^{*}=\min _{i \in \mathrm{jets}} \Delta \phi\left(\vec{p}_{\mathrm{T} i}, \vec{H}_{\mathrm{T}}^{\mathrm{miss}}+\vec{p}_{\mathrm{T} i}\right)
$$

The events in the search region do not have an isolated electron, muon, or track and satisfy the conditions that $n_{\text {jet }} \geq 1$, the largest jet $p_{\mathrm{T}}>100 \mathrm{GeV}, H_{\mathrm{T}}>200 \mathrm{GeV}, H_{\mathrm{T}}^{\text {miss }}>130 \mathrm{GeV}$, $H_{\mathrm{T}}^{\text {miss }} / E_{\mathrm{T}}^{\text {miss }}<1.25$ and $\Delta \phi_{\min }^{*}>0.5$. In addition, the events with $H_{\mathrm{T}}<800 \mathrm{GeV}$ have $\alpha_{\mathrm{T}}$ greater than a certain threshold between 0.52 and 0.65 depending on $H_{\mathrm{T}}$.

In the Run 1 analyses [7, 28-31], the events in the search region are generally required to have at least two jets with $p_{\mathrm{T}}$ greater than $100 \mathrm{GeV}$. The analysis in Ref. [4], on the other hand, catego- 
rizes the events that satisfy this requirement as symmetric and includes two additional categories: asymmetric and monojet. An event in the asymmetric category have one jet with $p_{\mathrm{T}}$ greater than $100 \mathrm{GeV}$ and one or more jets with $p_{\mathrm{T}}$ between $40 \mathrm{GeV}$ and $100 \mathrm{GeV}$. An event in the monojet category has one jets with $p_{\mathrm{T}}$ greater than $100 \mathrm{GeV}$ and no $2^{\text {nd }}$ jet with $p_{\mathrm{T}}$ greater than $40 \mathrm{GeV}$. The events in each of these three categories are further categorized in the bins of $n_{\mathrm{jet}}, n_{\mathrm{b}}, H_{\mathrm{T}}$, and $H_{\mathrm{T}}^{\text {miss }}$.

The SM background is predicted from the single-muon, double-muon, and single-photon CRs. The observed numbers of the events and the predictions, provided in each bin of $n_{\text {jet }}, n_{\mathrm{b}}$, and $H_{\mathrm{T}}$ in tables in Ref. [4], are consistent. A summary of the interpretations is shown in the next section.

\section{Interpretation}

This section summarizes interpretations of the results in the squark pair and gluino pair productions in simplified models for which diagrams are show in Fig 9, i.e., T2qq, T1tttt, T1bbbb, and T1qqqq. Refs. [1-3] include interpretations in other simplified models as well.

Figure 10 shows an interpretation of the results of the analysis summarized in Section 4 in T2qq. On the neutralino-squark mass plane, the upper limits on the production cross sections are shown as the color scale. The thick lines show the observed lower limits on the masses of neutralinos and squarks. The limits are shown for two cases: that both chiral states of all lightflavored squark have the same mass and that one chiral state of one light-flavored squark enters the model. The thick dashed lines show the expected lower mass limits, the limits that would be obtained if the predicted number of the events were actually observed. For the former case, the squark mass up to about $1260 \mathrm{GeV}$ and the neutralino mass up to about $580 \mathrm{GeV}$ are excluded. For the latter case, the squark and neutralino masses up to $600 \mathrm{GeV}$ and $300 \mathrm{GeV}$ are excluded.

Figures 11, 12, and 13 summarize observed and expected lower mass limits on the neutralinogluino mass planes in T1tttt, T1bbbb, and T1qqqq, respectively, placed by the analysis summarized in these proceedings. Figures similar to Fig. 10, i.e., showing the cross section limits as the color scale, for these models are provided in Refs. [1-4,32]. Figure 11 includes the limits placed by other
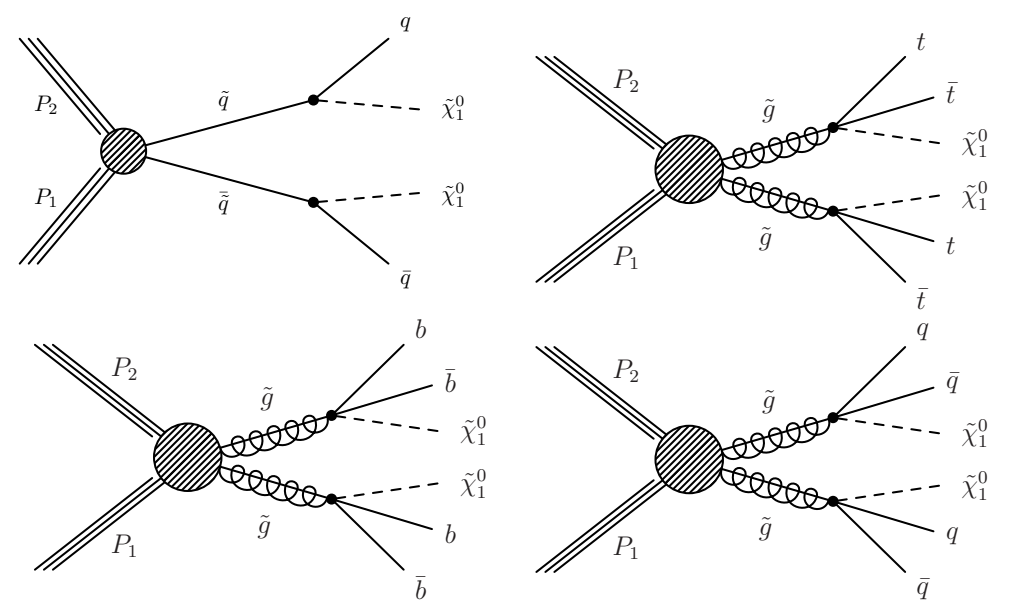

Figure 9: Diagrams for the gluino pair and squark pair productions in simplified models: T2qq (top left), T1ttt (top right), T1bbbb (bottom left), T1qqqq (bottom right). 
analyses with the data collected during the same period [33-36]. In each figure, a limit placed by an analysis with the $19 \mathrm{fb}^{-1}$ of data at $8 \mathrm{TeV}$ collected during Run 1 is also shown for a comparison $[20,37,38]$. The gluino mass up to $1750 \mathrm{GeV}$ in the limit of massless neutralinos and the neutralino mass up to $1125 \mathrm{GeV}$ in the case of the light gluinos are excluded. These limits are significantly raised from the Run 1 results.

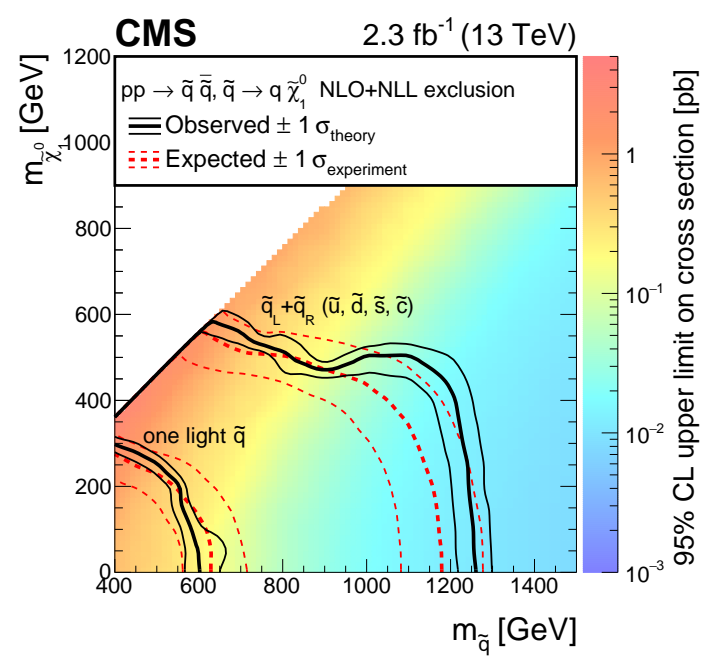

Figure 10: The limit on the production cross section of squark pairs and on the masses on the $\widetilde{\chi}^{0}-\widetilde{q}$ mass plane in T2qq for the case in which both chiral states of all light-flavored squark have the same mass and the case in which one chiral state of one light-flavored squark enters the model [2].

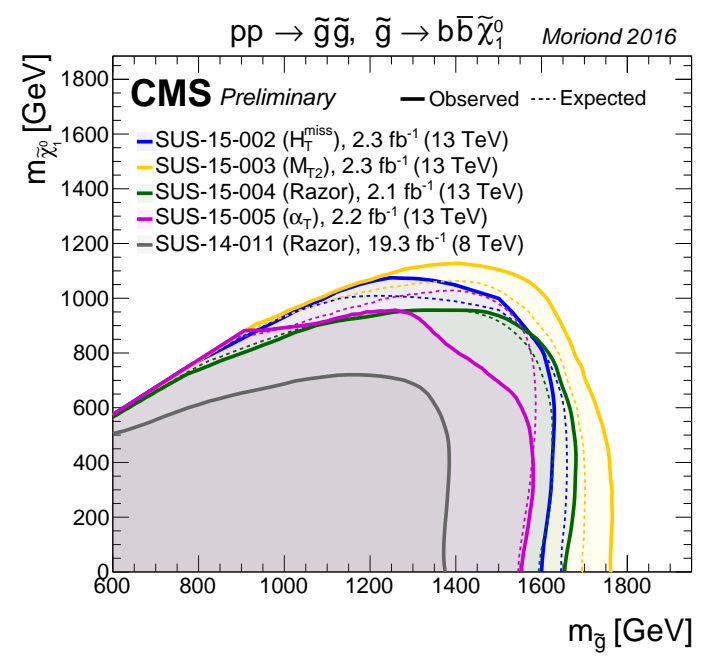

Figure 12: The limits on the $\widetilde{\chi}^{0}$ - $\widetilde{g}$ mass plane for T1bbbb placed by the four analyses summarized in these proceedings [1-4] and one analysis with Run 1 data [38].

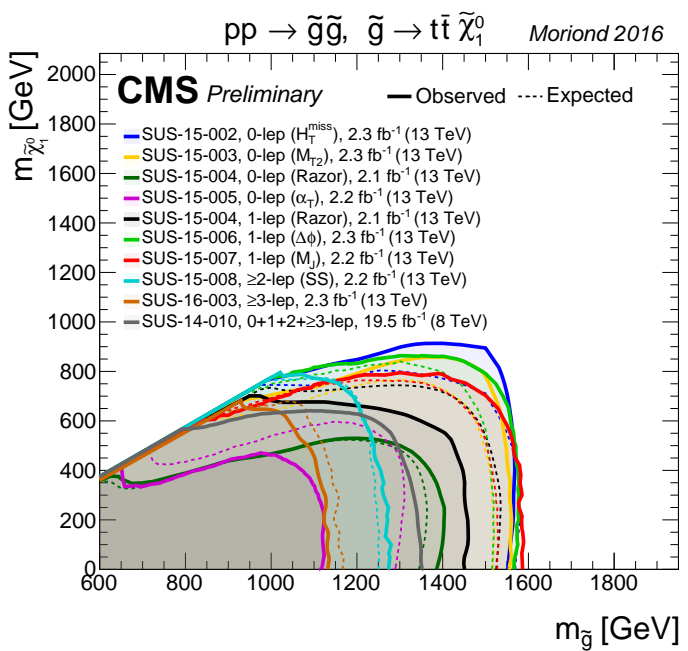

Figure 11: The limits on the $\widetilde{\chi}^{0}-\widetilde{\mathrm{g}}$ mass plane for $\mathrm{T} 1 \mathrm{tttt}$ placed by the four analyses summarized in these proceedings [1-4, 32], four other analyses with the data collected during the same period [33-36], and one analysis with Run 1 data [37]. The solid lines show the observed limits, and dashed lines show the expected limits.

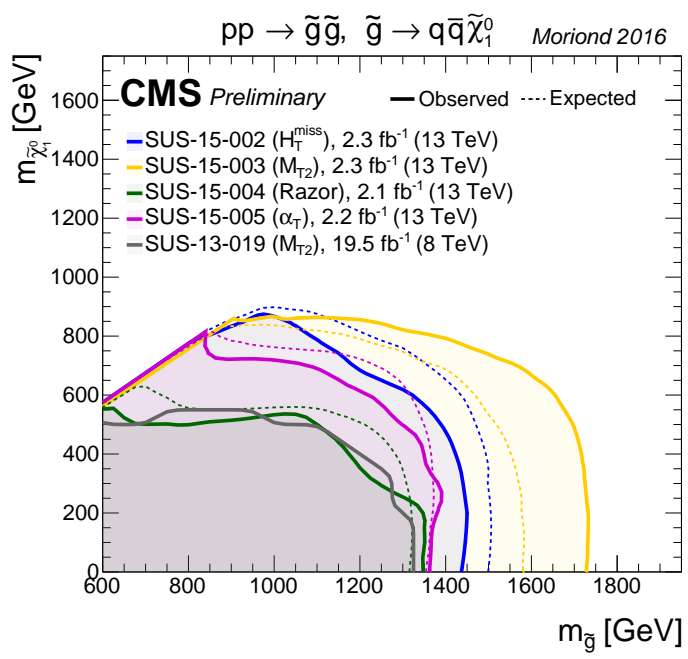

Figure 13: The limits on the $\tilde{\chi}^{0}$ - $\widetilde{g}$ mass plane for T1qqqq placed by the four analyses summarized in these proceedings [1-4] and one analysis with Run 1 data [20]. 


\section{Summary}

We have searched for pair productions of squarks and gluinos in hadronic channels in $2.3 \mathrm{fb}^{-1}$ of proton-proton collisions at $\sqrt{s}=13 \mathrm{TeV}$ collected with the CMS detector in 2015, the first year of LHC Run 2. The number of the event that we observed in the search region of each analysis was consistent with the standard model prediction. The limits on the production cross sections and masses of squarks and gluinos in simplified models have been significantly extended from the previous results. The data collection with the CMS detector in 2016 has been successful. We have released updates on the searches summarized in these proceedings with $12.9 \mathrm{fb}^{-1}$ of data collected in 2016 [39-41]. At the time of this writing, $28.8 \mathrm{fb}^{-1}$ of data have been collected. We are analyzing these data and continue to test the predictions of supersymmetric extensions of the standard models.

\section{References}

[1] CMS collaboration, Phys. Lett. B758 (2016) 152-180, [arXiv: 1602.06581].

[2] CMS collaboration, arXiv: 1603.04053.

[3] CMS collaboration, CMS-PAS-SUS-15-004.

[4] CMS collaboration, CMS-PAS-SUS-15-005.

[5] C. G. Lester and D. J. Summers, Phys. Lett. B463 (1999) 99-103, [arXiv: hep-ph/ 9906349 ].

[6] A. Barr, C. Lester et al., J. Phys. G29 (2003) 2343-2363, [arXiv: hep-ph/0304226].

[7] CMS collaboration, Phys. Lett. B698 (2011) 196-218, [arXiv:1101.1628].

[8] C. Rogan, arXiv:1006.2727.

[9] N. Arkani-Hamed, P. Schuster et al., arXiv: hep-ph/0703088.

[10] J. Alwall, P. Schuster et al., Phys. Rev. D79 (2009) 075020, [arXiv: 0810.3921 ].

[11] J. Alwall, M.-P. Le et al., Phys. Rev. D79 (2009) 015005, [arXiv: 0809.3264 ].

[12] D. S. M. Alves, E. Izaguirre et al., JHEP 10 (2011) 012, [arXiv: 1102. 5338].

[13] D. Alves, J. Phys. G39 (2012) 105005, [arXiv:1105.2838].

[14] CMS collaboration, Phys. Rev. D88 (2013) 052017, [arXiv: 1301.2175].

[15] T. Junk, Nucl. Instrum. Meth. A434 (1999) 435-443, [arXiv: hep-ex/ 9902006 ].

[16] A. L. Read, J. Phys. G28 (2002) 2693-2704.

[17] G. Cowan, K. Cranmer et al., Eur. Phys. J. C71 (2011) 1554, [arXiv: 1007.1727 ].

[18] CMS collaboration, JHEP 06 (2014) 055, [arXiv: 1402 .4770].

[19] CMS collaboration, Phys. Lett. B725 (2013) 243-270, [arXiv: 1305.2390].

[20] CMS collaboration, JHEP 05 (2015) 078, [arXiv: 1502.04358 ].

[21] CMS collaboration, JHEP 10 (2012) 018, [arXiv: 1207.1798].

[22] CMS collaboration, Phys. Rev. D93 (2016) 092009, [arXiv: 1602 . 02917].

[23] CMS collaboration, Phys. Rev. D92 (2015) 072006, [arXiv: 1507.02898].

[24] CMS collaboration, Phys. Rev. D91 (2015) 052018, [arXiv: 1502.00300 ].

[25] CMS collaboration, Phys. Rev. D90 (2014) 112001, [arXiv: 1405.3961$]$.

[26] CMS collaboration, Phys. Rev. Lett. 111 (2013) 081802, [arXiv:1212.6961].

[27] CMS collaboration, Phys. Rev. D85 (2012) 012004, [arXiv:1107.1279].

[28] CMS collaboration, Submitted to: Phys. Lett. B (2016), [arXiv:1605.08993].

[29] CMS collaboration, Eur. Phys. J. C73 (2013) 2568, [arXiv: 1303.2 2985].

[30] CMS collaboration, JHEP 01 (2013) 077, [arXiv: 1210.8115].

[31] CMS collaboration, Phys. Rev. Lett. 107 (2011) 221804, [arXiv: 1109.2352 ].

[32] CMS collaboration, CMS-PAS-SUS-16-004.

[33] CMS collaboration, CMS-PAS-SUS-15-006.

[34] CMS collaboration, Submitted to: JHEP (2016), [arXiv: 1605.04608].

[35] CMS collaboration, Eur. Phys. J. C76 (2016) 439, [arXiv:1605.03171].

[36] CMS collaboration, CMS-PAS-SUS-16-003.

[37] CMS collaboration, Phys. Lett. B745 (2015) 5-28, [arXiv: 1412.4109].

[38] CMS collaboration, CMS-PAS-SUS-14-011.

[39] CMS collaboration, CMS-PAS-SUS-16-014.

[40] CMS collaboration, CMS-PAS-SUS-16-015.

[41] CMS collaboration, CMS-PAS-SUS-16-016. 